\title{
Statistical Properties of Kumaraswamy-Generalized Exponentiated Exponential Distribution
}

\author{
B. E. Mohammed \\ Department of Mathematics, Faculty of Science, Al-Azhar University \\ Nasr City 11884, Cairo, Egypt
}

\begin{abstract}
In this paper, we present a new class of distributions called kumaraswamy Generalized Exponentiated Exponential Distribution, that is based upon the cumulative distribution function of Kumaraswamy (1980) distribution, which is more flexible and is a natural generalization of the exponential, Exponentiated Exponential and kumaraswamy Generalized exponential distributions as special cases found in literature. Also, the analytical shapes of the corresponding probability density function and hazard rate function are derived with graphical illustrations. Expressions for the $\mathrm{r}^{\text {th }}$ moments are calculated and the variation of the skewness and kurtosis measures is investigated. Likelihood estimators of the parameters are derived. Moreover, analysis of real data set, representing the breaking stress of carbon fibers, is conducted to demonstrate the usefulness of the proposed distribution.
\end{abstract}

\section{Keywords}

Kumaraswamy Distribution, Maximum likelihood estimation, Akaike information criterion, Baysian information criterion, Consistent Akaike Information Criteria, Kaplan-Meier estimator, likelihood ratio test, p-p plot.

\section{INTRODUCTION}

The Exponentiated Exponential (EE) distribution, a most attractive generalization of the exponential distribution, is defined as a particular case of Gompertz-Verhulst distribution function (see Ahuja and Nash (1967)). The EE distribution has been introdused and studied by Gupta and Kundu (1999, 2001 , 2003 , 2004, 2007). They observed that this distribution can be used in place of gamma and weibull distributions, since the two parameters of the gamma, wiebull, and EE distributions have increasing as well as decreasing hazard function depending on the value of the shape parameters, also they have a constant hazard function when the shape parameter is equal to one (Gupta and Kundu (1999)).

A random variable (rv) $X$ is said to have the $E E$ distribution if its cumulative distribution function (cdf) is defined by

$$
\begin{gathered}
F(x)=(1-\exp (-\lambda x))^{\alpha}, x>0, \alpha, \lambda \\
>0,
\end{gathered}
$$

and the probability density function (pdf) is given by

$f(x)=\alpha \lambda \exp (-\lambda x)(1-\exp (-\lambda x))^{\alpha-1}, x>0, \alpha, \lambda>0$,

where $\alpha$ and $\lambda$ are respectively shape and scale parameters. For different value of the shape parameters, the pdf can take different shapes.
Adding parameters to a well-established family of distributions are a time honored device for obtaining more flexible new families of distributions. Cordeiro and Castro (2011) defined the $\operatorname{cdf} F(x)$ and the pdf $f(x)$ of the Kumaraswamy generalized $(K w G)$ distribution by

$G(x)=1-\left\{1-F^{a}(x)\right\}^{b},-\infty<x<\infty$

and

$g(x)=a b f(x) F^{a-1}(x)\left\{1-F^{a}(x)\right\}^{b-1}$

respectively, where $f(x)=d F(x) / d x \quad$ and $a, b>0$ are additional shape parameters to the distribution F. Except for some special choices of the function $F(x)$, The density $g(x)$ will be difficult to deal with some generality. One major benefit of the $\mathrm{KwG}$ distribution is its ability of fitting skewed data that cannot be properly fitted by existing distributions. This fact was demonstrated recently by Cordeiro et al. (2010) who apply the Kumaraswamyweibull distribution to failure data.

A physical interpretation of the $K w G$ distribution given by Equations (1.3) and (1.4) (for a and b positive integers) is as follows. Consider that a system is formed by $b$ independent components and that each component is made up of a independent subcomponents. Suppose the system fails if any of the $\mathrm{b}$ components fails and that each component fails if all of the a subcomponents fail. Let $X_{j 1}, \ldots, X_{j a}$ denote the lifetimes of the subcomponents within the $j^{\text {th }}$ component, $j=1, \ldots, b$ having a common $\operatorname{cdf} G(x)$. Let $X_{1}$ denote the lifetime of the $j^{\text {th }}$ component, for $j=1, \ldots, b$ and let $X$ denote the lifetime of the entire system. Then, the cdf of $X$ is

$$
\begin{aligned}
\operatorname{Pr}(X \leq x)=1 & -\operatorname{Pr}\left(X_{1}>x, X_{2}>x, \ldots, X_{b}>x\right) \\
= & 1-\left\{\operatorname{Pr}\left(X_{1}>x\right)\right\}^{b}=1-\left\{1-\operatorname{Pr}\left(X_{1} \leq x\right)\right\}^{b} \\
= & 1-\left\{1-\operatorname{Pr}\left(X_{11} \leq x, X_{12} \leq x \ldots, X_{1 a} \leq x\right)\right\}^{b} \\
=1 & -\left\{1-\left[\operatorname{Pr}\left(X_{11} \leq x\right)\right]^{a}\right\}^{b}=1-\left\{1-F^{a}(x)\right\}^{b}
\end{aligned}
$$

So, it follows that the $K w G$ distribution given by (1.3) and (1.4) is precisely the time to failure distribution of the entire system.

In this paper, we introduce a new variant of KwG extended family of distribution by selecting in (1.3), the $E E$ cdf (1.1) whichyields

$G(x)=1-\left\{1-\left[(1-\exp (-\lambda x))^{\alpha}\right]^{a}\right\}^{b}, x>0, \alpha, a, b>0$. 
We shall write $X \sim K w G E E$ to denote an absolutely continuous rv $X$ possessing the $K w G$ extended $E E$ distribution with parameters $\lambda, \alpha, a, b$ and cdf given by (1.5).

The aim of this paper is to reveal some statistical properties of the $X \sim K w G E E$ distribution.

\section{DENSITY, MOMENTS AND QUANTILES}

The pdf of the $\operatorname{KwGEE}(\lambda, \alpha, a, b)$ distribution with $\operatorname{cdf}(1.4)$ is given by

$$
\begin{aligned}
g(x)= & a b \alpha \lambda \exp (-\lambda x)(1-\exp (-\lambda x))^{a \alpha-1} \\
& \left\{1-\left[(1-\exp (-\lambda x))^{\alpha}\right]^{a}\right\}^{b-1}, x>0 .
\end{aligned}
$$

\section{Remarks}

(i) For $a=1, b=1(1.4)$ and (2.1) reduce to the case of the $E E$ distribution.

(ii) For $a=1, b=1, \alpha=1,(1.4)$ and (2.1) reduce to the case of the exponential distribution.

(iii) For $\alpha=1,(1.4)$ and (2.1) reduce to the case of the kumaraswamy Generalized exponential distributions distribution (see Nadarajah et al. (2012)).

The following theorem gives simple conditions under which the pdf (2.1) is decreasing or unimodal.

\section{Theorem (1.2)}

The pdf of the KwGEE, given by (2.1), is decreasing or unimodal if $a \alpha-1<0$ or $a \alpha-1 \geq 0$ respectively.

\section{Proof}

The first derivative of $g(x)$ is given by

$$
\begin{aligned}
\grave{g}(x)=a b \alpha \lambda^{2} \exp (-\lambda x) & (1-\exp (-\lambda x))^{a \alpha-2}\{1 \\
- & {\left.\left[(1-\exp (-\lambda x))^{\alpha}\right]^{a}\right\}^{b-2} \eta(x), x>0, }
\end{aligned}
$$

where

$$
\begin{aligned}
\eta(x)=\{1-(1- & \left.\exp (-\lambda x))^{a \alpha}\right\}\{(a \alpha-1) \exp (-\lambda x) \\
& -(1-\exp (-\lambda x))\} \\
& -a \alpha(b \\
& -1) \exp (-\lambda x)(1-\exp (-\lambda x))^{a \alpha},
\end{aligned}
$$

the function $\eta(x)$ has no (one) zero on $(0, \infty)$ provided $\eta(0)=a \alpha-1 \leq 0(>0)$ that is, $g(x)$ has no (one) critical point provided $\eta(0) \leq 0(>0)$. Since $g(x)$ is nonnegative and $g(x)=0$ and, is decreasing (unimodal) provided $\eta(0) \leq$ $0(>0)$.

Fig. (2.1) below shows thepdfcurves for the KwGEE distribution for selected values of the parameters $\lambda, \alpha, a$ and $b$.



$\mathbf{x}$

(a) $\mathrm{a}=0.5, \alpha=2$, (bold), 0.9, 1(plain), 0.7, 1.3(point), $b=25$, $\lambda=0.1$

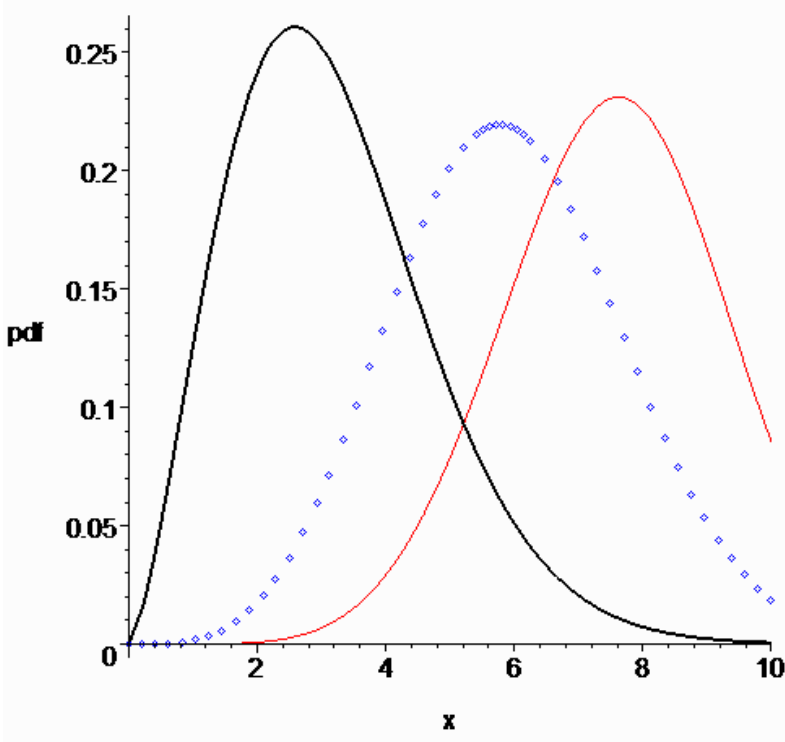

(b) $\mathrm{a}=0.5, \alpha=5$, (bold), 1, 2(plain), 1.5, 1.5(point), $b=40$, $\lambda=0.1$

Fig. 2.1: Thepdf $g(x)$ of the $K w G E E$ distribution for selected values of the parameters. In Fig.2. 1(a) $a \alpha<1$, showing that $g(x)$ is decreasing. In Fig.2. 1(b) $a \alpha>1$, showing that $g(x)$ is increasing-decreasing.

The $r^{t h}$ moment of the KwGEE distribution is given by

$$
\begin{aligned}
E\left(X^{r}\right)=r \int_{0}^{\infty} x^{r-1} \bar{G}(x) d x & =r \int_{0}^{\infty} x^{r-1} \\
& \left\{1-\left[(1-\exp (-\lambda x))^{\alpha}\right]^{a}\right\}^{b} d x,
\end{aligned}
$$


using the fact that

$$
\begin{aligned}
& \left\{1-\left[(1-\exp (-\lambda x))^{\alpha}\right]^{a}\right\}^{b} \\
& =\sum_{i=1}^{\infty}(-1)^{i}\left(\begin{array}{l}
b \\
i
\end{array}\right)(1-\exp (-\lambda x))^{a \alpha i},
\end{aligned}
$$

again,

$$
(1-\exp (-\lambda x))^{a \alpha i}=\sum_{j=1}^{\infty}(-1)^{j}\left(\begin{array}{c}
a \alpha i \\
j
\end{array}\right)(\exp (-\lambda x))^{j},
$$

then

$$
\begin{aligned}
E\left(X^{r}\right) & =r \sum_{i=1}^{\infty} \sum_{j=1}^{\infty}(-1)^{i+j}\left(\begin{array}{l}
b \\
i
\end{array}\right)\left(\begin{array}{c}
a \alpha i \\
j
\end{array}\right) \int_{0}^{\infty} x^{r-1}(\exp (-\lambda x))^{j} d x \\
& =\sum_{i=1}^{\infty} \sum_{j=1}^{\infty}(-1)^{i+j}\left(\begin{array}{c}
b \\
i
\end{array}\right)\left(\begin{array}{c}
a \alpha i \\
j
\end{array}\right) \frac{\Gamma(r+1)}{(j \lambda)^{r}}
\end{aligned}
$$

Since (2.2) is a convergent series for any $r \geq 0$, therefore putting $r=1$, we obtain the mean as

$$
E(X)=\sum_{i=1}^{\infty} \sum_{j=1}^{\infty}(-1)^{i+j}\left(\begin{array}{l}
b \\
i
\end{array}\right)\left(\begin{array}{c}
a \alpha i \\
j
\end{array}\right) \frac{\Gamma(2)}{(j \lambda)^{r}},
$$

and putting $r=2$, we obtain the second moment as

$$
E\left(X^{2}\right)=\sum_{i=1}^{\infty} \sum_{j=1}^{\infty}(-1)^{i+j}\left(\begin{array}{l}
b \\
i
\end{array}\right)\left(\begin{array}{c}
a \alpha i \\
j
\end{array}\right) \frac{\Gamma(3)}{(j \lambda)^{r}} .
$$

The $q^{\text {th }}$ quantile of the $K w G E E$ distribution is given by

$$
\begin{array}{r}
Q(u)=G^{-1}(q)=\frac{1}{\lambda \log \left[1-\left(1-(1-q)^{\frac{1}{b}}\right)^{\frac{1}{a \alpha}}\right]}, \\
0 \leq q \leq 1,
\end{array}
$$

where $G^{-1}($.$) is the inverse distribution function.$

In particular, the median of the KWGEE distribution is given by

$$
\operatorname{median}(X)=\frac{1}{\lambda \log \left[1-\left(1-\left(1-\frac{1}{2}\right)^{\frac{1}{b}}\right)^{\frac{1}{a \alpha}}\right.} .
$$

For $a=1, b=1$ we get the corresponding results for the $E E$ distribution.

\section{QUANTILE MEASURES}

To illustrate the effect of the shape parameters $\boldsymbol{a}$ and $\boldsymbol{b}$ on skewness and kurtosis of the new distribution, we consider measures based on quantiles. The shortcomings of the classical kurtosis measure are well known. There are many heavy-tailed distributions for which this measure is infinite, So, it becomes uninformative precisely when it needs to be. Indeed, our motivation to use quantile based measures stemmed from the non-existence of classical kurtosis for many generalized distributions.

The Bowley'sskewness (Kenney and Keeping, (1962)) is one of the earliest skewness measures defined by

$$
S k=\frac{Q(3 / 4)-2 Q(1 / 2)+Q(1 / 4)}{Q(3 / 4)-Q(1 / 4)},
$$

The Moors kurtosis (Moors, (1988)) based on cotiles is defined by

$$
K u=\frac{Q(7 / 8)-Q(5 / 8)-Q(3 / 8)+Q(1 / 8)}{Q(6 / 8)-Q(2 / 8)},
$$

where $Q($.$) represents the quantile function define in (2.3).$ The measures $\mathrm{Sk}$ and $\mathrm{Ku}$ are less sensitive to outliers and they exist even for distributions without moments. For symmetric unimodal distributions, positive kurtosis indicates heavy tails and peakedness relative to the normal distribution, whereas negative kurtosis indicates light tails and flatness. For the normal distribution, $S k=K u=0$.

In figures 3.1 and 3.2, we plot the measures Sk and $\mathrm{Ku}$ for the $K w G E E(0.1,0.5, a, b)$ distribution, as functions of $b$ (for fixed a) and as functions of a (for fixed $b$ ), respectively. These plots indicate that the Bowley skewness always decreases when an increases (for fixed b), and always increases when $b$ increases (for fixed a). On the other hand, the Moors kurtosis always decreases when a increases (for fixed b) and always increases when $b$ increases (for fixed a). So, these plots indicate that both measures can be very sensitive on these shape parameters, thus indicating the importance of the proposed distribution. 

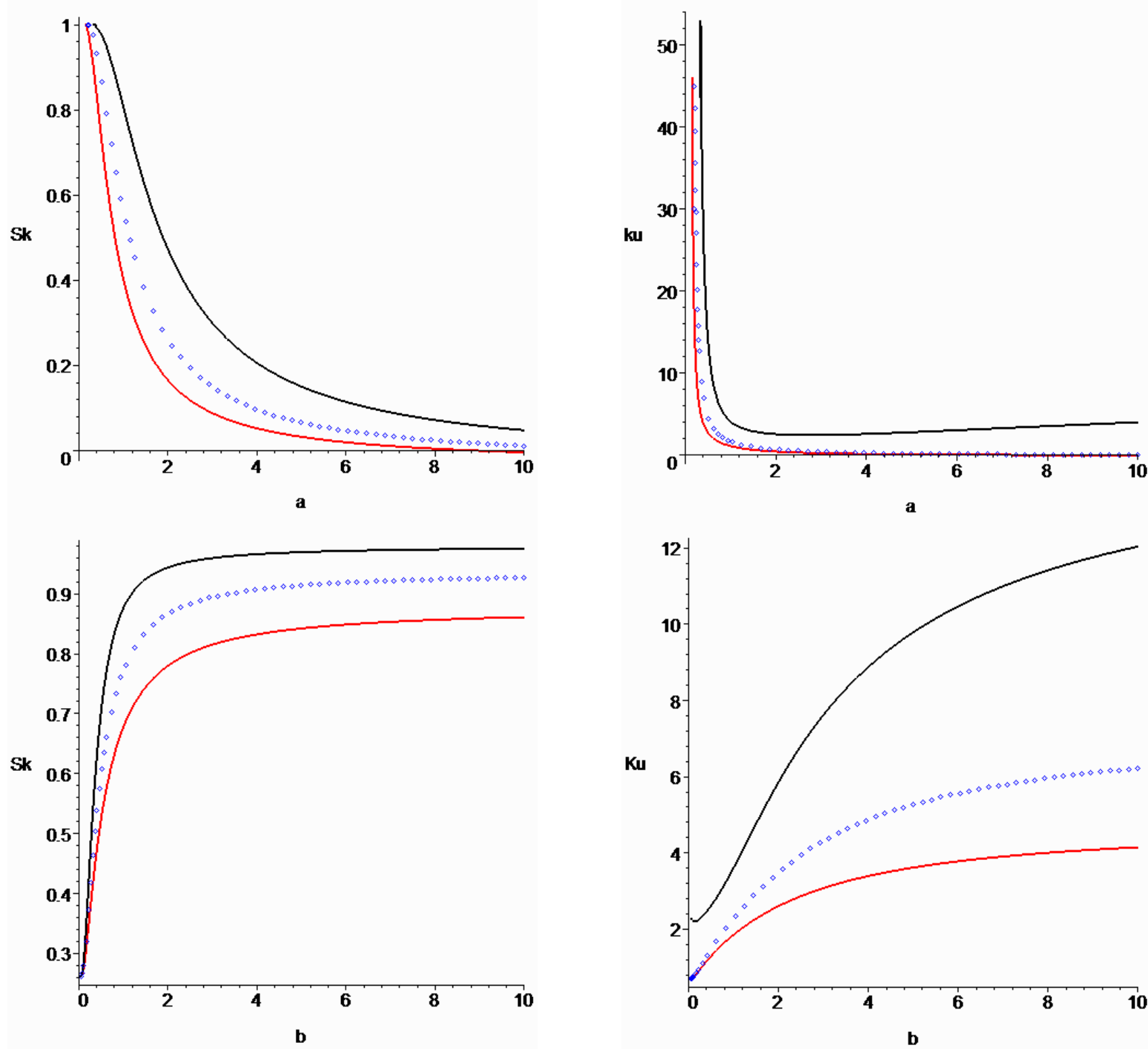

Fig. (3.1): The Bowleyskewness of the KwGEEdistribution as function of $b$ some values of $a$ and as function of $a$ for some values of $b$.

Fig. (3.2): The Moors kurtosis of the $K w G E E$ distribution as function of $b$ for some values of $a$ and as function of $a$ for some values of $b$.

\section{HAZARDRATE FUNCTION}

The hazard rate function (hrf) of the KwGEEdistribution is given by

$h(x)=\frac{a b \alpha \lambda \exp (-\lambda x)(1-\exp (-\lambda x))^{a \alpha--1}}{\left\{1-\left[(1-\exp (-\lambda x))^{\alpha}\right]^{a}\right\}}$.

Note that for all $b, \lambda$, we have

$$
\begin{gathered}
h(0)= \begin{cases}0 & \text { For } a \alpha>1 \\
\mathrm{ab} \alpha \lambda & \text { For } a \alpha=1 \\
\infty & \text { For } a \alpha<1\end{cases} \\
h(\infty)=\mathrm{b} \lambda \text { For all } a \alpha .
\end{gathered}
$$

The following theorem gives simple conditions under which hrf (4.1) is decreasing or increasing. 
For any $a, b, \lambda$ the hrf is an increasing if $a \alpha>1$, and it is a decreasing function if $a \alpha<1$. For $a \alpha=1$, it is constant (Fig. (4.1)).

\section{Remarks}

(i) For $a=1, b=1, \mathrm{~h}(\mathrm{x})$ is increasing if $\alpha>1$, decreasing if $\alpha<1$, and constant if $\alpha=1$, which is a well-known results for the $E E$ distribution (Gupta (2001)).

(ii) For $a=1, b=1, \lambda=1, \mathrm{~h}(\mathrm{x})$ is constant for all $\alpha$, which is a well-known results for the exponential distribution (Venkatesan and Sundaram (2011)).

Fig. (4.1) below shows the hrf curves for the KwGEE distribution for selected $\lambda, \alpha, a$ and $b$.



Fig. (4.1): $a \alpha<1$ (plain), $a \alpha=1$ (bold), $a \alpha>1$ (point),

$$
b=40, \lambda=0.1
$$

\section{STOCHASTIC ORDERING}

Stochastic ordering of positive continuous random variables is an important tool for judging the comparative behavior. We will recall some basic definitions. A random variable $\mathrm{X}$ is said to be smaller than a random variable $\mathrm{Y}$ in the

(i) stochastic order (denoted by $X \leq_{s t} Y$ ) if $\bar{F}_{X}(x)=\bar{F}_{Y}(x)$ for all $x$.

(ii) hazard rate order (denoted by $X \leq_{h r} Y$ ) if $h_{X}(x)=$ $h_{Y}(x)$ for all $x$.

(iii) likelihood ratio order (denoted by $X \leq_{l r} Y$ ) if $f_{X}(x) /$ $f_{Y}(x)$ decreases in $x$.

The following implications [see Ross ((1996), Chap. 9)] are well known:

$$
X \leq_{l r} Y\left(X \leq_{h r} Y, X \leq_{s t} Y\right) .
$$

The KWGEE distributions are ordered with respect to the strongest likelihood ratio ordering, as shown in the following theorem.

\section{Theorem (5.1)}

Let $X \sim \operatorname{KwGEE}\left(\lambda, \alpha, a, b_{1}\right)$ and $\quad Y \sim \operatorname{KwGEE}\left(\lambda, \alpha, a, b_{2}\right) . \quad$ If $b_{2}<b_{1}$ then

$$
X \leq_{l r} Y\left(X \leq_{h r} Y, X \leq_{s t} Y\right) .
$$

\section{Proof}

First note that

$$
\frac{g_{X}(x)}{g_{Y}(x)}=\frac{b_{1}\left\{1-(1-\exp (-\lambda x))^{a \alpha}\right\}^{b_{1}-1}}{b_{2}\left\{1-(1-\exp (-\lambda x))^{a \alpha}\right\}^{b_{2}-1}}
$$

Since, for $b_{2}<b_{1}$,

$$
\begin{aligned}
\frac{d}{d x} \frac{g_{X}(x)}{g_{Y}(x)}=a \alpha \lambda \frac{b_{1}}{b_{2}}\left(b_{2}-\right. & \left.b_{1}\right)(1-\exp (-\lambda x))^{a \alpha} \\
& \left\{1-(1-\exp (-\lambda x))^{a \alpha}\right\}^{b_{1}-1}
\end{aligned}
$$

$g_{X}(x) / g_{Y}(x)$ is decreasing in $x$; that is $X \leq_{l r} Y$.The remaining statements follow from the implications (5.1).

\section{ESTIMATION OF KwGEEDISTRIBUTION}

In this section, we determine the maximum likelihood estimates (MLEs) of the parameters $(\lambda, \alpha, a, b)$ of the KwGEE distribution. Suppose $X_{1}, X_{2}, \ldots, X_{n}$ is a random sample of size $\mathrm{n}$ from theKwGEE distribution. Then the likelihood function is given by

$$
\begin{array}{r}
\prod_{i=1}^{n} g_{X}\left(x_{i}\right)=\prod_{i=1}^{n} \mathrm{ab} \alpha \lambda \exp \left(-\lambda x_{i}\right)\left(1-\exp \left(-\lambda x_{i}\right)\right)^{a \alpha-1} \\
\left\{1-\left(1-\exp \left(-\lambda x_{i}\right)\right)^{a \alpha}\right\}^{b-1},
\end{array}
$$

and the log-likelihood function is given by

$$
\begin{aligned}
L=\log \left[\prod_{i=1}^{n} g_{X}\left(x_{i}\right)\right]= & n \log (a b \alpha \lambda)-\lambda \sum_{i=1}^{n} x_{i} \\
& +(a \alpha-1) \sum_{i=1}^{n} \log \left(1-\exp \left(-\lambda x_{i}\right)\right) \\
& +(b-1) \sum_{i=1}^{n} \log \left[1-\left(1-\exp \left(-\lambda x_{i}\right)\right)^{a \alpha}\right] .
\end{aligned}
$$

The estimates of the parameters maximize the likelihood function. Taking the partial derivatives of the log-likelihood function with respect to $\lambda, \alpha, a, b$ respectively and equalizing the obtained expressions to zero yields to likelihood equations.

$$
\begin{aligned}
\frac{\partial L}{\partial \lambda}= & \frac{n}{\lambda}-\sum_{i=1}^{n} x_{i}+(a \alpha-1) \sum_{i=1}^{n} \frac{x_{i} \exp \left(-\lambda x_{i}\right)}{\left(1-\exp \left(-\lambda x_{i}\right)\right)} \\
& \quad-(b-1) \sum_{i=1}^{n} \frac{\left.a \alpha x_{i} \exp \left(-\lambda x_{i}\right)\left(1-\exp \left(-\lambda x_{i}\right)\right)^{a \alpha-1}\right)}{1-\left(1-\exp \left(-\lambda x_{i}\right)\right)^{a \alpha}}, \\
\frac{\partial L}{\partial \alpha}= & \frac{n}{\alpha}+a \sum_{i=1}^{n} \log \left(1-\exp \left(-\lambda x_{i}\right)\right) \\
& -(b-1) \sum_{i=1}^{n} \frac{a x_{i}\left(1-\exp \left(-\lambda x_{i}\right)\right)^{a \alpha} \log \left(1-\exp \left(-\lambda x_{i}\right)\right)}{1-\left(1-\exp \left(-\lambda x_{i}\right)\right)^{a \alpha}},
\end{aligned}
$$




$$
\begin{aligned}
\frac{\partial L}{\partial b} & =\frac{n}{b}+\sum_{i=1}^{n} \log \left[1-\left(1-\exp \left(-\lambda x_{i}\right)\right)^{a \alpha}\right], \\
\frac{\partial L}{\partial a} & =\frac{n}{a}+\alpha \sum_{i=1}^{n} \log \left(1-\exp \left(-\lambda x_{i}\right)\right) \\
& -(b-1) \sum_{i=1}^{n} \frac{a x_{i}\left(1-\exp \left(-\lambda x_{i}\right)\right)^{a \alpha} \log \left(1-\exp \left(-\lambda x_{i}\right)\right)}{1-\left(1-\exp \left(-\lambda x_{i}\right)\right)^{a \alpha}} .
\end{aligned}
$$

The maximum likelihood estimates $(M L E s) \hat{\lambda}, \hat{\alpha}, \hat{a}, \hat{b}$ of the parameters $\lambda, \alpha, a, b$ are obtained numerically by solving the non-linear equations $\frac{\partial L}{\partial \lambda}=0, \frac{\partial L}{\partial \alpha}=0, \frac{\partial L}{\partial a}=0$, and $\frac{\partial L}{\partial b}=0$.

The likelihood ratio test will be used to test the null hypothesis $H_{0}: a=1, b=1$ ( $E E$ distribution). When $H_{0}$ is true, the deviance teststatistic $d_{n}=-2\{L(\tilde{\lambda}, \tilde{\alpha}, 1,1)-$ $L(\hat{\lambda}, \hat{\alpha}, \hat{a}, \hat{b})\}$, where $\tilde{\lambda}$, $\tilde{\alpha}$ are the MLEs of $\lambda$, $\alpha$ under $H_{0}: a=$ $1, b=1$, has approximately a chi-square distribution with 2 degree of freedom. $H_{0}$ is rejected at a significance level of $\alpha$ if $d_{n}>\chi_{2, \alpha}^{2}$.

In addition, for model selection, we use the Akiake Information Criterion $(A I C)$, the Bayesian Information Criterion $(B I C)$ and the Consistent Akaike Information Criteria $(C A I C)$ defined as:

$A I C=-2 \log$ likelihood $+2 q$

$B I C=-2 \log$ likelihood $+q \log (n)$

$C A I C=-2 \log$ likelihood $+\frac{2 q n}{n-q-1}$

where $q$ is the number of parameters in the model and $\mathrm{n}$ is the sample size. For more details about the AIC, BIC, and CAIC see Akiake (1969), Schwarz (1978), and Bozdogan (1987) respectively. The model with smaller $A I C, B I C$ and $C A I C$ is the one that better fits the data.

\section{APPLICATION}

In this section, we use a real data set to show that the KwGEE distribution can be a better model than one based on the EE and exponential distribution. We make a results comparison of the models fit. We consider an uncensored data set corresponding an uncensored data set from consisting of 100 observations on breaking stress of carbon fibers as discussed by Shams (2013). The data are:

$\begin{array}{lllllll}3.7 & 2.74 & 2.73 & 2.5 & 3.6 & 3.11 & 1.12 \\ 3.27 & 2.87 & 1.47 & 3.11 & 1.84 & 0.39 & 1.71 \\ 2.88 & 4.42 & 2.41 & 3.19 & 3.22 & 1.69 & 2.03 \\ 3.28 & 3.09 & 1.87 & 3.15 & 4.9 & 3.68 & 1.61 \\ 2.48 & 2.82 & 3.75 & 2.43 & 2.95 & 2.97 & 1.69 \\ 3.39 & 2.96 & 2.53 & 2.67 & 2.93 & 3.22 & 4.38 \\ 0.85 & 1.61 & 2.05 & 3.39 & 2.81 & 4.2 & 2.17 \\ 3.33 & 2.55 & 3.31 & 3.31 & 2.85 & 2.56 & 1.17 \\ 3.56 & 2.79 & 4.7 & 3.65 & 3.15 & 2.35 & 5.08 \\ 2.55 & 2.59 & 2.38 & 2.81 & 2.77 & 2.17 & 2.48 \\ 2.83 & 1.92 & 2.03 & 1.8 & 1.89 & 1.41 & 1.18 \\ 3.68 & 2.97 & 1.36 & 0.98 & 2.76 & 4.91 & 1.25 \\ 3.68 & 1.84 & 1.59 & 1.57 & 1.08 & 2.12 & 3.51 \\ 3.19 & 1.57 & 0.81 & 5.56 & 1.73 & 1.59 & 2.17 \\ 2 & 1.22 & & & & & \end{array}$

The following tablegives a comparison between the $M L E S$, log-likelihood, AIC, BIC and CAIC for the fitted KwGEE, EE and exponential distributions to the given data. The table shows small values of both $A I C, B I C$, and $C A I C$ which favour selecting the KWGEE distribution.

\begin{tabular}{|c|c|c|c|c|c|c|}
\hline Model & parameter & MLE & L & AIC & BIC & CAIC \\
\hline Exponential & $\lambda$ & 0.381476 & -196.371 & 394.742 & 397.347 & 394.783 \\
\hline \multirow{2}{*}{ EE } & $\lambda$ & 1.01317 & -146.182 & 296.365 & 301.575 & 296.488 \\
& $\alpha$ & 7.78824 & & & & \\
\hline \multirow{2}{*}{ KwGEE } & $\lambda$ & 0.110961 & & 290.637 & 301.057 & 291.058 \\
& $a$ & 4.95024 & \multirow{2}{*}{-141.318} & & & \\
& $\mathrm{~b}$ & 6.660032 & & & & \\
\hline
\end{tabular}


The results in the above table show that the fitted KwGEE distribution should be selected based on either the CAIC or $B I C$ or $A I C$ procedure.

For the given data, under $H_{0}, \quad L(\tilde{\lambda}, \tilde{\alpha}, 1,1)=-146.182$ thus $d_{n}=9.728>\chi_{2.0 .05}^{2}=5.991$,

therefore, we cannot accept the null hypothesis, i.e. The likelihood ratio test rejects the assumption that the EE model is sutable for the given data.

Also, $\quad$ let $H_{0}, \quad L(\tilde{\lambda}, 1,1,1)=-196.371$, (exponential distribution) thus

$d_{n}=110.106>\chi_{3.0 .05}^{2}=7.815$, therefore, we cannot accept the null hypothesis, i.e. The likelihood ratio test rejects the assumption that the exponential model is sutable for the given data.

Let $n$ be the total number of breaking stress of carbon fibers whose survival times, uncensored data, are available. Relabel the $n$ survival times in order of increasing magnitude such that $t_{(1)} \leq t_{(2)} \leq \cdots \leq t_{(n)}$. The Kaplan-Meier (1958) estimator (KME), also known as the product limit estimator, of a survival function is defined as

$$
\bar{G}_{n}(t)=\prod_{t: t_{(i)} \leq t}\left\{1-\frac{1}{n-r+1}\right\}, \quad t>0 .
$$

Figs. (7.1), (7.2) and (7.3) show, respectively, the p-p plot of the KME versus the fitted exponential, $E E$ and $K w G E E$ survival functions for the given data.



Fig. (7.1): p-p plot of KME versus fitted exponential survival function



Fig. (7.2): p-p plot of KME versus fitted EE survival function



Fig. (7.3): p-p plot of KME versus fitted $K w G E E$ survival function

Visually, the depicted points for fitted KwGEE survival function are very near the $45^{\circ}$ line, indicating very good fit as compared with the fitted $E E$ survival function.

Since $\hat{\lambda}=0.110961, \hat{\alpha}=4.95024, \hat{a}=0.660032, \quad$ and $\hat{b}=66.246$, then the estimated hrt $h(x)$ is as shown in the following figure. 


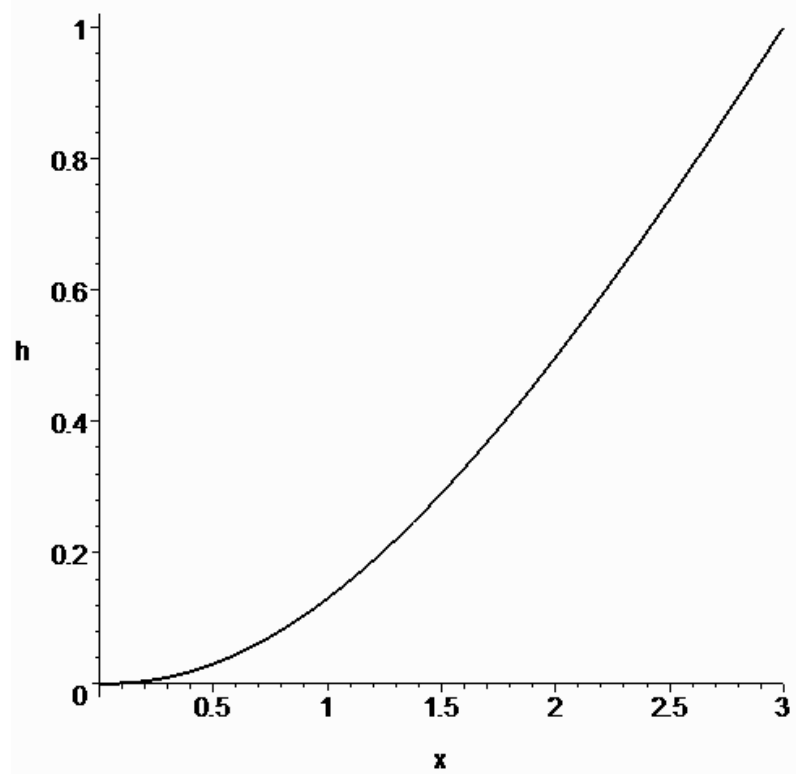

Fig. (7.4): The estimated hazard rate function of $K w G E E$ distribution based on observations the breaking stress of carbon fibers.

\section{CONCLUSION}

We note that for KwGEE distribution, the $A I C, B I C$ and $C A I C$ are smaller than the corresponding $A I C, B I C$ and $C A I C$ of the EE and exponential distributions. Also the fitted $K w G E E$ survival function indicates strong linear relationship between the empirical and fitted survival functions comparing with the fitted $E E$ and exponential survival functions. All these results lead us to the real data set was analyzed and the $K W G E E$ has provided a good fit for the given data and was more appropriate model.

\section{ACKNOWLEDGMENTS}

The author would like to thank the referees for their kind effort and precious time for reviewing this manuscript.

\section{REFERENCES}

[1] Ahuja, J. C. and Nash, S. W. 1967 The generalized Gompertz-Verhulst family of distributions. Sankhya, Series A. 29, 141-156.

[2] Akaike, H. 1969 Fitting Autoregressive Models for Prediction. Annals of the Institute of Statistical Mathematics, 21, 243-247.

[3] Bozdogan, H. 1987 Model selection and Akaike's information criterion (AIC): The general theory and its analytical extensions. Psychometrika, 52, 345-370.

[4] Cordeiro, G. M. and Castro, M. 2011 A new family of generalized distributions. Journal of Statistical Computation and Simulation 81, 883-898.
[5] Cordeiro, G. M., Ortega, E. M. and Nadarajah, S. 2010 The Kumaraswamy Weibull distribution. Journal of the Franklin Institute 347, 1399-1429.

[6] Gupta, R. D. and Kundu, D. 1999 Generalized Exponential Distributions. Australian and New Zealand Journal of Statistics. 41, 173-188.

[7] Gupta, R. D. 2001 Exponentiated Exponential Family; An Alternative to Gamma and Weibull. Biometrical Journal. 33, 117-130.

[8] Gupta, R. D. and Kundu, D. 2001 Generalized Exponential Distributions: Different Methods of Estimation. Journal of Statistical Computation and Simulation. 69, 315-338.

[9] Gupta, R. D. and Kundu, D. 2003 Discriminating Between the Weibull and the GE Distributions. Computational Statistics and Data Analysis. 43, 179-196.

[10] Gupta, R. D. and Kundu, D. 2004 Discriminating Between the Gamma and Generalized Exponential Distributions. Journal of Statistical Computation and Simulation. 74, 107-121.

[11] Gupta, R. D. and Kundu, D. 2007 Generalized exponential distribution: existing methods and recent developments. Journal of the Statistical Planning and Inference. 137, 3537--3547.

[12] Kaplan, E. L. and Meier, P. (1958). Nonparametric estimation from incomplete observations. Journal of the American Statistical Association, 53, 457--481.

[13] Kenney, J. F. and Keeping, E. S. 1962 Mathematics of Statistics (3rd ed.), Part 1. New Jersey.

[14] Kumaraswamy, P. 1980 Generalized probability density function for double bounded random processes. Journal of Hydrology. 462, 79-- 88.

[15] Moors, J. J. 1988 A quantile alternative for kurtosis. Journal of the Royal Statistical Society D, 37, 25-32.

[16] Nadarajah, S., Cordeiro, G. M., and Ortega. E. M. 2012 General results for the Kumaraswamy-G distribution. Journal of Statistical Computation and Simulation. 82, 951--979.

[17] Ross, S. M. 1996 Stochastic processes, 2nd ed., New York: Wiley.

[18] Schwarz, G. 1978 Estimating the Dimension of a Model. Annals of Statistics 6, 461-464.

[19] Venkatesan, P. and Sundaram, N. 2011 Exponentiated exponential models for survival data. Indian Journal of Science and Technology. 4, 923-930. 